\title{
Bradfords «Brussels-Effect» als Gedankenstütze in der Diskussion um ein institutionelles Rahmenabkommen zwischen der Schweiz und der Euro-
} päischen Union

\section{Einleitung}

ANU BRADFORDS These von der Existenz eines BrusselsEffect prägt im angelsächsischen Raum den wissenschaftlichen Diskurs über die geopolitische Rolle der Europäischen Union. Danach übt die EU einen überproportional grossen Teil an extraterritorialer Macht via marktzugangsbezogene Regulatorien aus. In der Schweiz ist der Effekt nicht unbekannt, denn ein Diskurs über die Marktmacht der Union ist für die Schweiz unumgänglich. Das liegt insbesondere an der Struktur der schweizerischen Volkswirtschaft, die stark durch Handel mit Unternehmen aus der EU geprägt ist und in faktischer Abhängigkeit vom Zugang zum europäischen Markt funktioniert. Den Brussels-Effect in BRADFORDS Form aber expliziter miteinzubeziehen, könnte für die (politische) Diskussion gerade jetzt Mehrwerte liefern, wo der Bundesrat entschieden hat, das institutionelle Abkommen zwischen der Schweiz und der EU nicht zu unterzeichnen. Der vorliegende Beitrag erörtert, um dies zu verdeutlichen, verschiedene Aspekte des Verhältnisses zwischen der Schweiz und der EU im Licht von BRADFORDS Theorie.

II. Bradfords These vom Brussels-Effect

Zitiervorschlag:

RAPHAEL DUMMERMUTH, Bradfords «Brussels-Effect» als Gedankenstütze in der Diskussion um ein institutionelles Rahmenabkommen zwischen der Schweiz und der Europäischen Union, sui generis 2021, S. 245

MLaw Raphael Dummermuth, Diplomassistent am Lehrstuhl für Zivilrecht I an der Universität Freiburg. Der Autor dankt Prof. Dr. iur. Alexandra Jungo, ordentliche Professorin für Zivilrecht an der Universität Freiburg, MLaw Sofie Steller, Diplomassistentin am Lehrstuhl für Europarecht und europäisches Migrationsrecht an der Universität Freiburg, und BLaw Dominic von Moos.

URL: sui-generis.ch/188

DOI: https://doi.org/10.21257/sg.188

Dieses Werk ist lizenziert unter einer Creative Commons Namensnennung - Weitergabe unter gleichen Bedingungen 4.0 International Lizenz. 


\section{Einleitung}

1 ANU BRADFORDS ${ }^{1}$ These von der Existenz eines BrusselsEffect $^{2}$ prägt im angelsächsischen Raum den wissenschaftlichen Diskurs über die geopolitische Rolle der Europäischen Union. ${ }^{3}$ Danach übt die EU einen überproportional grossen Teil an extraterritorialer Macht via marktzugangsbezogene Regulatorien aus. In der Schweiz ist der Effekt nicht unbekannt, ${ }^{4}$ denn ein Diskurs über die Marktmacht der Union ist für die Schweiz unumgänglich. Das liegt insbesondere an der Struktur der schweizerischen Volkswirtschaft, die stark durch Handel mit Unternehmen aus der EU geprägt ist und in faktischer Abhängigkeit vom Zugang zum europäischen Markt funktioniert. ${ }^{5}$ Den Brussels-Effect in BRADFORDS Form aber expliziter miteinzubeziehen, könnte für die (politische) Diskussion gerade jetzt Mehrwerte liefern, wo der Bundesrat entschieden hat, das institutionelle Abkommen zwischen der Schweiz und der EU nicht zu unterzeichnen. ${ }^{6}$ Der vorliegende Beitrag deutet, um dies zu verdeutlichen, verschiedene Aspekte des Verhältnisses zwischen der Schweiz und der EU im Licht von BRADFORDS Theorie.

\section{Bradfords These vom Brussels-Effect}

2 Die Diskussion über den geopolitischen Einfluss der Union konzentriert sich ausserhalb des europäischen Kontinents nicht selten auf deren militärische Schwäche, das schwerfällige Handeln der europäischen Institutionen, sowie die scheinbare Unmöglichkeit der EU-Mitgliedstaaten, international als geeinte Kraft aufzutreten und mit «einer Stimme» zu sprechen. ${ }^{7}$ Europa wird nicht selten als «aging and declining power ${ }^{8}$ gesehen. ${ }^{9}$ BRADFORD

1 Prof. Dr. ANU BRADFORD, LL.M., Henry L. Moses Professor of Law and International Organization, Columbia Law School.

2 ANU BRADFORD, The Brussels Effect: How the European Union Rules the World, New York 2020 (zit. BRADFORD 2020); ANU BRADFORD, The Brussels Effect, Northwestern University Law Review 107/2012, S.1ff. (zit. BRADFORD 2012).

3 Vgl. SUSAN EMMENEGGER, Extraterritoriality in Financial Regulation, in: Weber/Stoffel/Chenaux/Sethe (Hrsg.), Aktuelle Herausforderungen des Gesellschafts- und Finanzmarktrechts, Festschrift für Hans Caspar von der Crone zum 6o. Geburtstag, Zürich 2017, S. $391 \mathrm{ff.}$; vgl. weiter ORLA LYNSKEy, The Foundations of EU Data Protection Law, Oxford 2015, S. 42; PAUL M. SCHWARZ, The EU-U.S. Privacy Collision: A Turn to Institutions and Procedures, Harvard Law Review 2012/2013, S.1967.

4 Als eine der wenigen EMMENEGGER (Fn.3), S. 392.

5 Vgl. hierzu die Darstellung von MICHAEL HAHN, Je t'aime...Moi non plus : Rechtsfragen des Verhältnisses Schweiz-EU, ZBJV 157/2021, S. $77 \mathrm{ff}$.

6 Medienmitteilung des Bundesrates vom 26. Mai 2021 (Das Institutionelle Abkommen Schweiz-EU wird nicht abgeschlossen).

7 BRADFORD 2012 (Fn. 2), S. 2.

8 BRADFORD 2020 (Fn. 2), Introduction, S. XII.

9 So etwa aus dem angelsächsischen Raum WALTER RUSSEL MEAD, The incredible Shrinking Europe: The Continents Grand Union Project hält dieser Ansicht die faktische Fähigkeit der EU entgegen, globale Märkte zu regulieren, indem sie über das unionsrechtliche Sekundärrecht ${ }^{10}$ Vorschriften über die Herstellung, den Anbau, den Vertrieb und die Anpreisung von Produkten aufstellt oder beispielsweise wettbewerbsrechtliche und datenschutzrechtliche Bestimmungen über das Handeln Privater erlässt. ${ }^{11}$ Dies ruft nach BRADFORDS Einordnung einen «involuntary incentive» hervor: Im Rahmen einer regulatorischen Vereinheitlichung passen Unternehmen ihr gesamtes Markthandeln den Vorgaben des unionsrechtlichen Sekundärrechts an, und zwar auch in Staaten, die nicht im territorialen Geltungsbereich des Unionsrechts liegen. Entsprechend verhalten sich multinationale Unternehmen auch ausserhalb der EU unionsrechtskonform. ${ }^{12}$

Nach BRADFORD übt die Union damit eine Form der extra- 3 territorialen Macht aus, ohne hierfür jedoch auf das Tätigwerden von internationalen Institutionen angewiesen zu sein oder sich internationaler Kooperation mit anderen geopolitischen Entitäten zu bedienen.13

Zur Verdeutlichung dieser Theorie sind sowohl Abgren- 4 zungen als auch Unterscheidungen vorzunehmen. Erstens kann abgrenzend festgehalten werden: Der hier beschriebene Regulierungseffekt durch das sekundäre Unionsrecht muss von anderen globalen Vereinheitlichungstendenzen unterschieden werden, die auf bi- oder multilateralen Übereinkommen basieren. Ebenso ist festzuhalten, dass ein Wille der regulierenden Behörden bzw. Akteure kein Tatbestandsmerkmalbzw. keine Voraussetzung für die Verwirklichung des Brussels-Effect darstellt.

Zweitens ist eine Grundunterscheidung elementar für die 5 Effect-Theorie: Der Brussels-Effect de facto tritt dann auf, wenn global agierende Unternehmen bzw. Unternehmensgruppen ihr Handeln auf europäische Regulierungen bzw. europäische Standards ausrichten. Vom Brussels-Effect dejure hingegen spricht BRADFORD dann, wenn Unternehmen bzw. unternehmensnahe Interessengruppen in ihren Sitzstaaten für die Europäisierung der dort geltenden Rechtsordnung Lobbying betreiben oder auf andere Weise politischen Druck ausüben. Dieser «Europäisierungsdruck» ist hierbei typischerweise mit der

is Fading, Wall Street Journal vom 12. Februar 2019; WALTER RUSSEL MEAD, Europe's Challenge is Decline, not Trump, Wall Street Journal vom 18. Februar 2019; STEPHEN WALT, The Coming Erosion of the European Union, Foreign Policy vom 18. August 2014; TONY BARBER, The Decline of Europe is a Global Concern, The Financial Times vom 21. Dezember 2015.

10 Zum Begriff des europarechtlichen Sekundärrechts MATTHIAS OESCH, Europarecht, Band I, Grundlagen, Institutionen, Verhältnis Schweiz-EU, Bern 2015, N 415.

11 BRADFORD 2012 (Fn. 2), S. 2

12 BRADFORD 2012 (Fn. 2), S. 2; BRADFORD 2020 (Fn. 2), S. 2.

13 BRADFORD 2020 (Fn. 2), Introduction, S. XII. 
Motivation verknüpft, gegenüber Unternehmen mit Sitz in einem Mitgliedstaat der Union keinen Nachteil zu erleiden. Das beabsichtigte Resultat des Brussels-Effect de jure ist in der Schweiz gemeinhin als «autonomer Nachvollzug» bekannt und wird verschiedentlich auch der wissenschaftlichen Untersuchung zugeführt. ${ }^{14}$ Im Kontext dieses Artikels wird zwischen den Effekten de facto und de jure nicht von zwei Seiten derselben Medaille ausgegangen; der Brussels-Effect de jure ist m.E. gesondert zu behandeln. Die folgenden Ausführungen beziehen sich deshalb ausschliesslich auf den Brussels-Effect defacto (fortan schlicht Brussels-Effect).

Für die Lesenden weiter klärend wirken wohl die Voraus setzungen, die BRADFORD für das Eintreten des Effekts formuliert: Eine genügende Grösse des durch die Regulierungen kontrollierten Marktes (nachfolgend a); eine genügende «institutionelle Kapazität» zur Regulierung (b); ein unelastisches bzw. immobiles Ziel der Regulierung (c); eine fehlende ökonomische Rentabilität der Differenzierung bzw. «Aufsplittung» der Produktion der vom Unternehmen angebotenen Güter oder Dienstleistungen (d); und schliesslich die Tatsache, dass die untersuchten Regulierungen strikter sind als die Regelungen anderer vergleichbar attraktiver Märkte (e). Sind diese Voraussetzungen erfüllt, so die Theorie BRADFORDS, stellt sich auf globaler Ebene der dargelegte Brussels-Effect ein: Private Unternehmen halten sich in ihrem Marktverhalten global an die Normen des unionsrechtlichen Sekundärrechts. Das Unionsrecht erhält damit durch rein unilaterales Vorgehen eine extraterritoriale Wirkung von einer gewissen Tragweite.

\section{Voraussetzung für das Eintreten des Brussels-Effect}

a) Grösse des kontrollierten Martkes

7 Zur Ausübung geopolitischer Macht im Bereich des internationalen Handels wird die Grösse des kontrollierten Markts gemeinhin als zentrales Erfordernis genannt. ${ }^{15}$

14 Vgl. etwa ASTRID EPINEY, 20Jahre Institut für Europarecht der Universität Freiburg, Ein Beitrag zu Forschung, Lehre und Weiterbildung im Europarecht in der Schweiz, in: Forum Europarecht (Hrsg.), Die Schweiz und die europäische Integration: 20Jahre Institut für Europarecht / La Suisse et l'intégration européenne: 20 ans de l'Institut de droit européen, Freiburg 2015, S.11ff.; MATTHIAS OESCH, Die Europäisierung des schweizerischen Rechts, in: Cottier (Hrsg.), Die Europakompatibilität des schweizerischen Wirtschaftsrechts: Konvergenz und Divergenz, ZSR-Beiheft 50 2012, S.13 ff.; FRANCESCO MAIANI, Lost in translation: euro-compatibility, legal security, and the autonomous implementation of EU law in Switzerland, ELR 2013, S. 29 ff.; MARC AMSTUTZ, Normative Kompatibilitäten: Zum Begriff der Europakompatibilität und seiner Funktion im Schweizer Privatrecht, SJER 2004/2005, S. 235; THOMAS COTTIER, Zwischen Skylla und Charybdis: Die Rezeption des europäischen Wirtschaftsrechts in der Schweiz, EuZW 2012, S. 849.

15 Vgl. umfassend DANIEL W. DREZNER, Globalization, Harmonization, and Competition: The Different Pathways to Policy Convergence, Journal of European Public Policy 2005, S. $841 \mathrm{ff}$.; DANIEL W.
BRADFORDS Ansatz unterscheidet sich von herkömmlichen Analysen des geopolitischen Einflusses ${ }^{16}$ von dominanten Märkten dadurch, dass sie die schiere Grösse einer Volkswirtschaft als wichtig, aber nicht als ausschliesslichen Faktor darstellt. Während die EU einen Binnenmarkt mit mehr als 448 Millionen im globalen Vergleich überdurchschnittlich kaufkräftigen Konsument:innen darstellt und ein BIP von rund 17 Milliarden Euro aufweist, ${ }^{17}$ ist für den geopolitischen Erfolg der EU nach der Brussels-Effect-Theorie nicht dies der ausschlaggebende Faktor. Den Unterschied machen vielmehr weitere strukturelle Faktoren bzw. institutionelle Grundfunktionen der EU18 sowie der Wohlstand und die Konsumfreudigkeit der Konsument:innen im Europäischen Binnenmarkt. ${ }^{19}$

Da der Markt in der EU nicht nur einen zahlenmässig ver- 8 gleichsweise grossen Binnenmarkt darstellt, ${ }^{20}$ sondern auch einen überdurchschnittlich hohen Anteil an sehr kaufkräftigen Konsument:innen bietet, ${ }^{21}$ werden sich jene multinationalen Unternehmen, welche die oben zusammengefasste Abwägung vornehmen, mit grosser Wahrscheinlichkeit für den Eintritt in den EU-Markt entscheiden, ${ }^{22}$ was BRADFORD als eine Form regulatorischer Gravitation beschreibt. Ein Unternehmen aus einem Drittstaat wird zur Frage, ob es sich wirtschaftlich lohnt, mit Marktteilnehmer:innen aus der EU Geschäftsbeziehungen einzugehen, eine Abwägung vornehmen zwischen den Kosten, die eine Anpassung an das Unionsrecht mit sich bringt, und dem durch den Marktzugang prognostizierbaren Mehrwert. ${ }^{23}$

\section{b) Institutionelle Kapazität zur Durchsetzung strikter Regulatorien}

Nach BRADFORD hat zu den beschriebenen zahlenmäs- 9 sigen Faktoren ein zentrales Element dazuzutreten: die institutionelle Kapazität, Regulatorien zu implementieren und, wo nötig, durch Zwang durchzusetzen. ${ }^{24}$ Damit der Brussels-Effect seine Wirkung entfaltet, ist es entsprechend notwendig, dass die EU einerseits Regelungen von

DREZNER, All Politics is Global: Explaining International Regulato ry Regimes, Princeton NJ 2008.

16 Vgl. DREZNER, Globalization (Fn.15), S. 841.

17 Vgl. Internationaler Währungsfonds (IWF), Jahresbericht 2019: Unsere vernetze Welt.

18 BRADFORD 2012 (Fn. 2), S. 11 ff.; BRADFORD 2020 (Fn. 2), S. 27 f.

19 BRADFORD 2020 (Fn. 2), S. 27.

20 Central Intelligence Ageny (CIA), The World Factbook, European Union, Economy.

21 Zur zentralen Rolle der Kaufkraft der Konsument:innen DAVID VOGEL / ROBERT A. KAGAN, Dynamics of Regulatory Change: How Globalization Affects National Regulatory Policy. An Introduction, Berkley 2004, S. 6; BRADFORD 2020 (Fn. 2), S. 27.

22 BRADFORD 2012 (Fn. 2), S. 12; mit aktualisierten Zahlen BRADFORD 2020 (Fn. 2), S. $27 \mathrm{f}$.

23 BRADFORD 2020 (Fn. 2), S. 26.

24 BRADFORD 2012 (Fn. 2), S. 12; vgl. RAYA KARDASHEVA, Package Deals in EU Politics, American Journal of Political Science 2013, S. $858 \mathrm{ff}$. 
einer genügenden Normendichte in Kraft setzt. Die Nichtbeachtung dieser Regelungen muss jedoch im territorialen Anwendungsbereich des Unionsrechts für Private spürbare Konsequenzen haben; Marktteilnehmende, die sich (im Fall der EU) sekundärrechtswidrig verhalten, müssen vom gemeinsamen Markt ausgeschlossen werden können. Diesen regulatorisch-institutionellen Druck nennt BRADFORD Regulatory Capacity. ${ }^{25}$ Neben dieser regulatorischen «Infrastruktur» ist für die Wirkung strikter Regelungen für den Marktzugang auch der politische Wille notwendig, solche Regelungen zu schaffen, in Kraft zu setzen und in der Folge durchzusetzen.

\section{c) Unelastisches Ziel der Regulatorien}

10 Voraussetzung für die effektive Wirkung des Brussels-Effect ist weiter, dass das Objekt dieser Regelungen bis zu einem gewissen Grad immobil ist. Die verbraucherrechtlichen Regulierungen der EU, seien sie datenschutzrechtliche Bestimmungen, Minimalstandards für die Produktesicherheit oder Vorschriften aus dem Bereich des Lebensmittelmarkts, beziehen sich auf ein «regulatory target», das, anders etwa als Kapital, ${ }^{26}$ in einem hohen Grad immobil ist. ${ }^{27}$ Damit verstärkt nach der Analyse BRADFORDS der Fokus der regulatorischen Tätigkeit der Union auf den Bereich des Konsumentenschutzes entsprechend den Brussels-Effect. ${ }^{28}$

\section{d) Fehlende Rentabilität der Differenzierung der Produktion}

11 Damit unionsrechtliche Regulatorien aber auch im Handel zwischen Unternehmen mit Sitz in Drittstaaten und Konsument:innen bzw. anderen Unternehmen in Drittstaaten faktische Geltung erlangen, also wirklich «globale Wirkung» entfalten, ist ein weiterer Schritt notwendig: Die Unternehmen passen nicht nur ihr Verhalten gegenüber Konsument:innen oder Unternehmen innerhalb der EU dem Unionsrecht an, sondern ihre gesamte, globale Unternehmenstätigkeit.

12 Die dafür notwendige Unteilbarkeit des Produktionsprozesses («non-divisibility»29) eines Unternehmens kann verschiedene Gründe haben. Im Vordergrund steht eine mangelnde ökonomische Rentabilität der Aufteilung von Produktionsprozessen. Regelmässig werden Unternehmen bei einer entsprechenden Abwägung zum Schluss kommen, dass die Aufrechterhaltung eines einzigen Produktionsprozesses unter Einhaltung unionsrechtlicher

\footnotetext{
25 BRADFORD 2012 (Fn. 2), S. 12; BRADFORD 2020 (Fn. 2), S. 31; weiter gehend MANUELE CITI / MORGENS JUSTENSEN, Measuring and EXplaining Regulatory Reform in the EU: A Time-Series Analysis of Eight Sectors, 1984-2012, SSRN 2013, S.1984 ff.

26 BRADFORD 2020 (Fn. 2), S. 49.

27 BRADFORD 2012 (Fn. 2), S. 17; BRADFORD 2020 (Fn. 2), S. $48 \mathrm{ff}$.

28 BRADFORD 2012 (Fn. 2), S. 17; BRADFORD 2020 (Fn. 2), S. 53.

29 BRADFORD 2020 (Fn. 2), S. 53.
}

Vorschriften unter ökonomischen Aspekten dem Aufbau eines zweiten Produktionsprozesses vorzuziehen ist. ${ }^{30}$ In gleicher Weise als unteilbar haben Produktionsprozesse zu gelten, welche aus rechtlichen oder technischen Gründen nicht in zwei (oder mehr) Prozesse aufteilbar sind.

\section{e) Striktere Regulierung als die Regulierungen anderer attraktiver Märkte}

Die Ausführungen über die rechtliche sowie die techni- 13 sche Unmöglichkeit der «Aufsplittung» von Produktionsprozessen machen deutlich, dass ein Phänomen wie der Brussels-Effect so lange virulent bleibt, wie keine andere geopolitische Entität von gleichem ökonomischem Einfluss strengere Regulatorien erlässt. So sind etwa Szenarien denkbar, in welchen sich Unternehmen zugunsten strikter regulierender Staaten und gegen EU-Regulatorien entscheiden würden. Damit würde sich der Brussels-Effect abschwächen. Dies würde sich mit grosser Wahrscheinlichkeit schleichend und branchenspezifisch ankündigen.

\section{Notwendigkeit einer Diskussion}

Obgleich BRADFORD selbst die nun folgende Unterschei- 14 dung nicht macht, darf nach der hier vertretenen Auffassung nicht übersehen werden, dass sich durchaus ein Kreis von Staaten bzw. Weltregionen identifizieren lässt, die vom beschriebenen Effekt stärker betroffen bzw. beeinflusst werden als andere. Zur Kategorisierung von intensiver beeinflussten und weniger stark beeinflussten Staaten ist primär auf die Struktur einer Volkswirtschaft abzustellen; eine Volkswirtschaft mit einer hohen Dichte an Unternehmen, deren Produkte bzw. Dienstleistungen hauptsächlich Absatz im territorialen Geltungsbereich des europäischen Sekundärrechts finden, wird den Brussels-Effect stärker zu spüren bekommen als eine Volkswirtschaft, die weniger Handelsbeziehungen mit Marktteilnehmenden aus dem EU-Raum unterhält.

Die schweizerische Volkswirtschaft wird vom Handel mit 15 der EU entscheidend geprägt. 52\% der Exporte schweizerischer Unternehmen gehen in die EU; kein Markt ist für die schweizerische Industrie gleich wichtig wie der EU-Markt. Pro Arbeitstag werden zwischen der Union und der Schweiz Waren im Wert von einer Milliarde Franken ausgetauscht. ${ }^{31}$ Gewisse Wirtschaftszweige sind vom EU-Markt jedoch deutlich stärker abhängig, so liegt beim Schweizer Käse der Exportanteil in die EU bei 80\%.32

\footnotetext{
30 BRADFORD 2012 (Fn. 2), S.17 f.; BRADFORD 2020 (Fn. 2), S. 54.

31 Mission der Schweiz bei der Europäischen Union, Wirtschaft und Handel - eine wichtige Partnerschaft.

32 Economiesuisse, Annahme der Kündigungsinitiative bedeutet das Ende des bilateralen Wegs. Jedes einzelne bilaterale Abkommen bringt der Schweiz Vorteile, 13. September 2019.
} 

scheint es nun angebracht, die Schweiz als eines, wenn nicht das Paradebeispiel für eine vom Brussels-Effect geprägte Volkswirtschaft zu bezeichnen. Damit wird klar, dass der extraterritoriale Einfluss des europäischen Sekundärrechts auf die Schweiz - abgebildet im Konzept des Brussels-Effect - ein undiskutabler Fakt ist. Für Rechtsunterworfene in der Schweiz besteht mit anderen Worten ein Bereich, in welchem EU-Recht weder via Staatsvertrag, noch via autonomen Nachvollzug gilt, sondern ausschliesslich aufgrund von ökonomischem Druck. ${ }^{33}$ Dass dieser ökonomische Druck jedoch signifikanten Einfluss auf die nationale schweizerische Gesetzgebung sowie auf das schweizerische Staatsvertragsrecht zeitigt, soll in der Folge näher dargestellt werden.

\section{Das institutionelle Rahmenabkommen}

\section{Bilateralismus als Ausgangslage}

17 Im Licht der nun dargelegten Theorie und den dazugehörigen Anwendungsfällen aus der schweizerischen Rechtsordnung kann die Erkenntnis gewonnen werden, dass die Effect-Theorie für die Schweiz im Grunde genommen eine alltägliche Lebensrealität abbildet. ${ }^{34}$ Gleichzeitig kann aber gerade die Systematisierung, die BRADFORD vornimmt, für die hierzulange herrschende Diskussionslage einordnend bzw. strukturierend wirken. So kann der Effect, angewandt auf spezifische rechtliche oder politische Fragen, als Gedankenstütze dienen, indem er in die Analyse der Europäisierung der schweizerischen Rechtsordnung miteinbezogen wird. Zu diesem Zweck drängt es sich auf, im Folgenden die relevanten staatsvertraglichen Rahmenbedingungen stark zusammengefasst wiederzugeben.

Zunächst zu nennen sind die bilateralen Markzugangsabkommen zwischen der EU und der Schweiz, die für Marktteilnehmer:innen aus der Schweiz und der EU den Zugang zum jeweils anderen Markt garantieren. ${ }^{35}$ Unter-

33 Im Lichte dessen unklar erscheint die Aussage von HANSJÖRG SEILER, Auswirkungen desEU-Rechts auf Nicht-EU-Mitglieder («de facto Mitgliedschaft» der Schweiz und Liechtensteins?), XVI. Treffen der obersten Verwaltungsgerichtshöfe Österreichs, Deutschlands, des Fürstentums Liechtenstein und der Schweiz vom 18./19. September 2008, S. 12.

$34 \mathrm{Zu}$ den «Grundstrukturen» der Europäisierung des schweizerischen Rechts vgl. ANDREAS HEINEMANN, Rechtliche Transplantate zwischen Europäischer Union und der Schweiz, in: Fahrländer/ Heinzmann (Hrsg.), Europäisierung der schweizerischen Rechtsordnung, Zürich 2013, S.13ff.

35 Weitergehend ASTRID EPINEY / BEATE METZ / BENEDIKT PIRKER, Zur Parallelität der Rechtsentwicklung in der EU und in der Schweiz: ein Beitrag zur rechtlichen Tragweite der "Bilateralen Abkommen", Forum Europarecht Band 24, Zürich 2012, S. 98 f.; ANDREAS GLASER, schieden werden hierbei gemeinhin zwei «Pakete» von Verträgen, die durch eine «Guillotineklausel» ${ }^{36}$ verbundenen «Bilateralen I» aus dem Jahr 1999 und die (vorallem politisch eine Gruppe bildenden) «Bilateralen II», wobei «Schengen» und «Dublin» verknüpft sind, die übrigen Abkommen jedoch nicht. ${ }^{37}$ Die Bilateralen Abkommen stellen in Bezug auf ihren materiellen Regelungsbereich sektoriell eng begrenzte völkerrechtliche Verträge dar, die eine Integration der schweizerischen Volkswirtschaft in den europäischen Binnenmarkt zum Ziel haben. ${ }^{38}$ Diese «Teilintegration» geschieht durch unterschiedliche Herangehensweisen: Erstens stellen einige der bilateralen Abkommen Kooperationsverträge dar, die in Bezug auf gewisse EU-Programme die Teilnahme und die Zusammenarbeit der Schweiz garantieren. Zweitens existieren, was etwa den Personen- und den Landverkehr betrifft, Harmonisierungs- und Liberalisierungsverträge, die eine Angleichung der geltenden Regulatorien festlegen. Drittens wird in Bezug auf eine Reihe der bilateralen Verträge (etwa die Abkommen über die schweizerische Teilnahme am Schengen/Dublin-System) von Integrationsverträgen gesprochen. ${ }^{39}$

\section{Konzept eines institutionellen Rahmenabkommens}

Das Anliegen, einen Teil der völkerrechtlichen Verträge 19 zwischen der Europäischen Union und der Schweiz durch einen Rahmenvertrag zu verknüpfen, findet sich bereits im Integrationsbericht des Bundesrates von $1988 .{ }^{40}$ Bereits in Anbetracht der damaligen politischen Lage wünschte sich der Bundesrat eine «Schaffung von Strukturen und organisatorischen Vorkehrungen für eine privilegierte sektorielle Zusammenarbeit». ${ }^{41}$ Damit

Umsetzung und Durchführung des Rechts der Bilateralen Verträge in der Schweiz, in: Glaser/Langer (Hrsg.), Die Verfassungsdynamik der europäischen Integration und demokratische Partizipation, Zürich et al. 2015, S.133; HANSJÖRG SEILER, Einfluss des europäischen Rechts und der europäischen Rechtsprechung auf die schweizerische Rechtspflege, ZBJV 2014, S. 265; HANSJÖRG SEILER, Dynamik und Statik in der Rechtsprechung des Bundesgerichts zum EURecht?, in: Glaser/Langer (Hrsg.), Die Verfassungsdynamik der europäischen Integration und demokratische Partizipation, Zürich et al. 2015, S.77; CHRISTINE KADDOUS, Die Zusammenarbeit zwischen der EU und der Schweiz, in: Hatje/Müller-Graff (Hrsg.), Die Verfassungsdynamik der europäischen Integration und demokratische Partizipation, Erfahrungen und Perspektiven in Österreich und der Schweiz, Zürich et al. 2015, S. 21.

36 oEsCH, Europarecht Band I (Fn.10), N899.

37 OESCH, Europarecht Band I(Fn. 10), N901; zum Ganzen weiter PAULLUKAS GOOD, Die Schengen-Assoziierung der Schweiz, St. Gallen 2010.

38 OESCH, Europarecht Band I (Fn.10), N894.

39 EPINEY/METZ/PIRKER, Parallelität der Rechtsentwicklung (Fn. 35), S. 98 .

40 Bericht vom 24. August 1988 über die Stellung der Schweiz im europäischen Integrationsprozess (BBl1988 III 249), S. 347 f. (zit. Integrationsbericht).

41 Integrationsbericht(Fn. 40), S. $347 \mathrm{f}$. 
sollte eine grösstmögliche Mitwirkung der Schweiz am europäischen Integrationsprozess in einem sektoriellen Modus sichergestellt werden. Insbesondere genannt wird dabei die Idee des Aufbaus eines Systems «frühzeitiger Information und Abstimmung».42 Die politischen Aussichten auf den Abschluss eines solchen vertiefenden, institutionalisierenden Vertragssystems bewertet der Bundesrat damals als «günstig». 43

Dass die EU im Grundsatz eine vertiefte institutionalisierte Zusammenarbeit mit der Schweiz ebenso als wünschenswert erachtet, zeigen verschiedene Schlussfolgerungen bzw. Erklärungen des Rates sowie des Europäischen Parlamentes, gemäss welchen eine Weiterführung und Vertiefung des bilateralen Weges auch eine Institutionalisierung der Beziehungen zwischen der Schweiz und der EU anvisiert werden müsse. Mit Blick auf den Abschluss weiterer Abkommen herrscht in den europäischen Institutionen die Ansicht, weitere bilaterale Verträge seien ohne den Abschluss und das Inkrafttreten eines institutionellen Abkommens nicht denkbar. ${ }^{44}$

21 Wie die Weiterführung dieser Entwicklung angesichts des jüngst gefällten bundesrätlichen Entscheides aussehen wird, ist nicht absehbar. Wird vom verworfenen Entwurf ausgegangen, wäre ein institutionelles Abkommen als Weiterführung des bilateralen Wegs zu sehen. ${ }^{45}$ Ein Abkommen dieser Art würde sodann eine institutionell-konzeptionelle «Klammer» um einige der sektorspezifischen Abkommen bilden, indem für eine Reihe der geltenden Marktzugangsabkommen übergreifend gemeinsame Fragen geklärt würden. Ebenso würde ein Rahmenabkommen die konzeptuelle Basis für weitere bilaterale Übereinkommen zwischen denselben Parteien bilden und stellte damit das Fundament für die Weiterführung des bilateralen Wegs dar, indem «dynamische

42 Integrationsbericht (Fn. 40), S. 347.

43 Integrationsbericht (Fn. 40), S. 349.

44 Zum Ganzen: Rat der Europäischen Union, Schlussfolgerung des Rates zu einem homogenen erweiterten Binnenmarkt und den Beziehungen der EU zu nicht der EU angehörenden westeuropäischen Ländern vom 16. Dezember 2014 (16583/14), Rz. 44; Entschliessung des Europäischen Parlamentes vom 9. September $2015 \mathrm{zu}$ dem The ma EWR-Schweiz: Hindernisse bei der umfassenden Verwirklichung des Binnenmarktes (2017/C 316/19), S.16; vgl. OESCH, Europarecht Band I (Fn. 10), N956 ff.

45 Eidgenössisches Departement für auswärtige Angelegenheiten (EDA), Entwurf vom 23. November 2018 für ein Institutionelles Rahmenabkommen (Abkommen zur Erleichterung der bilateralen Beziehungen zwischen der Europäischen Union und der Schweizerischen Eidgenossenschaft in den Bereichen des Binnenmarkts, an denen die Schweiz teilnimmt; fortan E-InstA); vgl. zur Notwendigkeit der institutionellen Reform und der Dynamisierung der Verträge тномAS соттіER et al., Die Rechtsbeziehungen der Schweiz und der Europäischen Union, Bern 2014, S. 576; MATTHIAS OESCH / GABRIEL SPECK, Das geplante institutionelle Abkommen SchweizEU und der EuGH, in: Epiney/Hehemann (Hrsg.), Schweizerisches Jahrbuch für Europarecht 2016/2017, Bern et al. 2017, S. 252 ff.
Aktualisierungen» der Marktzugangsabkommen ermöglicht würden. ${ }^{46}$ Was dies im Detail zu bedeuten hat, kann bis zu einem gewissen Grad wiederum der verworfene Entwurf aufzeigen.

Zunächst gilt das verworfene Abkommen gem. Art. 2 E- 22 InstA für fünf sektorielle Bereiche: freier Personenverkehr, Luftverkehr, Schienen- und Strassenverkehr, technische Handelshemmnisse und landwirtschaftliche Erzeugnisse. Hernach ist das Abkommen als Rahmenordnung nach Art. 2 E-InstA auf sämtliche neu abzuschliessende Marktzugangsübereinkommen anwendbar. Eine automatische Rechtsübernahme sieht das Abkommen dabei aber nicht vor; die Schweiz hätte nach Art.10 bzw. Art.14 EInstA die Möglichkeit gehabt, gewisse Regelungen nicht zu übernehmen, wobei allerdings Ausgleichsmassnahmen möglich geblieben wären. Der Umfang bzw. der Inhalt der Ausgleichsmassnahmen würden jedoch, anders als unter der gegenwärtigen Rechtslage, nicht einseitig durch die EU bestimmt, sondern durch ein unabhängiges Schiedsgericht festgelegt (Art. 10 Abs. 6 EInstA) und wären auch nach ihrem Inkrafttreten noch gerichtlich überprüfbar (Art. 10 Abs. 7 E-InstA). ${ }^{47}$

Ebenso wird in Art. 9 E-InstA ein Streitbeilegungsverfah- 23 ren bei unterschiedlichen Rechtsauffassungen zwischen den Parteien in Bezug auf die Auslegung der Verträge und des Inst A selbst vereinbart. ${ }^{48}$ Hierfür wurde ein System erdacht, welches weiterhin ohne jede Form eines «Vorabentscheidungsverfahrens» über die Auslegung der Abkommensinhalte auskommt. Dem Bundesgericht kommt in diesem Konzept seine herkömmliche Rolle als letztinstanzliches Gericht zu. Hierbei werden die schweizerischen Gerichte dazu verpflichtet, die Rechtsprechung des EuGH zur Auslegung der Abkommen «laufend» zu übernehmen, was jedoch keiner Zeitenwende in der Rechtsanwendung gleichkäme, sondern im Gegenteil weitestgehend der bundesgerichtlichen Praxis entspricht. ${ }^{49} \mathrm{Bei}$ Streitigkeiten über die Auslegung der Verträge kommt es gemäss Protokoll 3 zum E-InstA zu einem Verfahren vor einem Schiedsgericht, falls Verhandlungen über die Auslegung im Gemischten Ausschuss scheitern. Das Schiedsgericht ist nach Art. 10 Abs. 3 EInstA und Protokoll 3 Art. III 9 dazu verpflichtet, Auslegungsfragen, die sich

46 Vgl. EDA, Institutionelles Abkommen Schweiz-EU: Das Wichtigste in Kürze, 7. Dezember 2018.

47 THOMAS COTTIER, Die Souveränität und das institutionelle Rahmenabkommen, SJZ 2019, S. 345 .

48 ASTRID EPINEY / SIAN AFFOLTER, das Institutionelle Abkommen und die Unionsbürgerrichtlinie, Jusletter vom 11. März 2019, S. 30; THOMAS COTTIER, Der Rechtsschutz im Rahmenabkommen SchweizEU, in: Epiney/Hehemann/Zlatescu (Hrsg.), Jahrbuch Europarecht 2018/2019, Bern et al. 2019, S. 362.

49 COTTIER, Souveränität (Fn. 47), S. 352. 
auf binnenmarktrechtliche Begriffe des europäischen Rechts beziehen, dem EuGH vorzulegen. 50

\section{Einordnung des E-InstA unter Zuhilfenahme des Brussels-Effect}

a) Ausgangslage: Theoretische Einordnung der Bilateralen Verträge

Der Bundesrat spricht sich bereits in seinem Integrationsbericht aus dem Jahr 1988 aus Gründen der volkswirtschaftlichen Notwendigkeit für eine weitergehende Kooperation mit der Union aus und hält fest, dass «die Qualität des Produktionsstandorts und Arbeitsplatzes Schweiz [...] massgeblich davon abhängen [wird], wie weit es uns gelingt, unsere Rechtssetzungeuropafreundlich zu gestalten [...]. Unser Ziel muss sein, in Bereichen von grenzüberschreitender Bedeutung (und nur dort) eine grösstmögliche Vereinbarkeit unserer Rechtsvorschriften mit denjenigen unserer europäischen Partner zu sichern.» ${ }^{51}$

Die schweizerische Politik der Europakompatibilität, wie sie der Integrationsbericht 1988 propagiert, nimmt damit BRADFORDS Analyse in ihren Auswirkungen bereits vorneweg, da sie u.A. ${ }^{52}$ auf der Erkenntnis beruht, dass für die schweizerische Volkswirtschaft eine unterbleibende oder mangelhafte Angleichung an das Unionsrecht in verschiedenen Bereichen zu markanten Wettbewerbsnachteilen für private Marktteilnehmer:innen führen würde. Das Bestreben zur Europäisierung entspricht als schweizerische Rechtssetzungsmaxime damit einer zutreffenden Analyse des Einflusses des Brussels-Effect auf die Schweiz, bevor diese Theorie überhaupt abstrakt formuliert wurde. Die schweizerischen Institutionen werden insofern in der Europapolitik also bereits seit längerem,

50 Zum Ganzen STEPHAN BREITENMOSER / SIMON HIRSBRUNNER, Der Entwurf für ein Institutionelles Rahmenabkommen zwischen der Schweiz und der EU: offene Fragen im Schnittpunkt zwischen Europa- und Völkerrecht, in: Epiney/Zlatescu (Hrsg.), Schweizerisches Jahrbuch für Europarecht 2019/2020, Bern et al. 2020; COTTIER, Souveränität(Fn. 47), S. 352; COTTIER, Rechtsschutz (Fn. 48), S.362; kritisch zur Funktion dieses Schiedsgerichts CARL BAUDENBACHER, Rechtsgutachten zur Streitentscheidungsregelung des InstA zu Handen der Kommssion des Nationalrates für Wirtschaft und Abgaben (WAK) vom 6. Februar 2019, sowie HANSUELI SCHÖCHLI, Dient das Schiedsgericht nur der Schweizer Psychohygiene?, NZZ vom 13. Dezember 2018, S. 25.

51 Integrationsbericht (Fn. 40), S. 380; vgl. weiter Bericht vom 29. November 1993 über die Aussenpolitik der Schweiz in den 90er Jahren (BBl 1994I153); eingehend PHILIPP w Yss, Europakompatibilität und Gesetzgebungsverfahren im Bund, AJP 2001, S. $717 \mathrm{ff}$.

52 Neben einem präventiven Ausgleich der marktbezogenen Nachteile ist ausdrücklich zu erwähnen, dass die Politik der Europaverträglichkeit zumindest zu Beginn der Neunzigerjahre auch das Ziel verfolgte, eine möglichst europakompatible Ausrichtung der schweizerischen Rechtsordnung zu bewahren, um für die Schweiz alle «integrationspolitischen Optionen, insb. ein möglicher EWRoder EG-Beitritt», offenzuhalten; vgl. MATTHIAS OESCH, Die Europäisierung des schweizerischen Rechts, Working Paper No 2011/05 Oktober 2011/70, S.29. gewissermassen non sequitur, durch die Überzeugung von der Existenz eines Brussels-Effect geleitet. Eine konsequent verfolgte Angleichung der schweizerischen Rechtsordnung an das Unionsrecht sollte die voraussehbaren Marktnachteile für private Akteure wo immer möglich vorbeugen. ${ }^{53}$ Die Europakompatibilität wird aus dieser Erkenntnis heraus zu einem Grundsatz der Rechtsetzung erhoben, welcher «die schweizerische Rechtsordnung als politisches Leitmotiv in ihrer ganzen Breite durchdringt». ${ }^{54}$ Bestärkt wurde die Schweiz in ihrer Politik der Europakompatibilität «faute de mieux» ${ }^{55}$ durch das EWR-Nein der schweizerischen Stimmbevölkerung im Dezember 1992. ${ }^{56}$

Die eben dargelegte grundsätzliche Erkenntnis schlägt 26 sich auch nach dem Integrationsbericht von 1988 und dem EWR-Nein von 1992 in verschiedenen Formen des politischen Handelns der Schweiz nieder; einerseits durch den sog. autonomen Nachvollzug, ${ }^{57}$ wo die Programme SWISSLEX und EUROLEX entworfen wurden, andererseits in völkerrechtlichen Verträgen mit der Union, in denen eine sektorielle Integration der Schweiz in den europäischen Binnenmarkt ermöglicht wurde. ${ }^{58}$

Als gezielter Ausgleich der «Nachteile des Abseitsste- 27 hens» ${ }^{59}$ und damit als Resultat einer schweizerischen Reaktion auf den festgestellten Brussels-Effect sind also auch die verschiedenen aussenpolitischen europapolitischen Massnahmen der Schweiz ab 1988 zu lesen. Dies

53 oEsCH, Working Paper (Fn. 52), S. 29.

54 OESCH, Working Paper (Fn. 52), S. 29; mit Verweis auf DANIEL THÜRER, Europaverträglichkeit als Rechtsargument. Zu den Wegen und Möglichkeiten schweizerischer Rechtsanpassung an die neue Integrationsdynamik der Europäischen Gemeinschaft in: Haller/ Müller/Kölz/Thürer (Hrsg.), Im Dienst an der Gesellschaft, Festschrift für Dietrich Schindler zum 65. Geburtstag, Basel et al. 1989, S. 561 .

55 OESCH, Europarecht Band I (Fn. 10), N892.

56 OESCH, Europarecht Band I (Fn. 10), N 892; vgl. zur historischen Aufarbeitung URS ALTERMATT, Die Schweiz in Europa: Antithese, Modell oder Biotop?, Frauenfeld et al. 2011; JUSTYNIA BRONSKA, Die Schweiz in Europa: Mittendrin, doch aussen vor? Auswirkungen eines EU-Beitritts im Kontext der Erfahrungen Österreichs, Marburg 2009; FRITZ BREUSS / THOMAS COTTIER / PETER-CHRISTIAN MÜLLER-GRAFF, Die Schweiz im europäischen Integrationsprozess, Basel 2008; THOMAS COTTIER, Die Schweiz und Europa: Herausforderungen im Vierten Kreis der Integration, in: Neuhaus (Hrsg.), Europa zu Beginn des 21. Jahrhunderts, Erlangen 2014, S. $141 \mathrm{ff}$.; JAKOB KELLENBERGER, Wo liegt die Schweiz?, Zürich 2014.

57 Zum Begriff etwa EPINEY (Fn.14), S.11 ff.; OESCH (Fn.14), S.13 ff.; MAIANI (Fn.14), S. 29 ff.; AMSTUTZ (Fn.14), S. 235; COTTIER (Fn.14), S. 849; Botschaft vom 24. Februar 1993 über das Folgeprogramm nach der Ablehnung des EWR-Abkommens (BBl1993 I 805); HEINRICH KOLLER, Der Schweizerische Gesetzgeber vor der internationalen Herausforderung: Erfahrungen mit «EUROLEX» - «SWISSLEX»«GATTLEX», ZBI 1994, S. 250 ff.; ROGER MALLEPELL, Der Einfluss des Gemeinschaftsrechts auf die schweizerische Gesetzgebung 1993-1995, Bern 1999, S.18ff.; vgl. zum Ganzen OESCH, Working Paper (Fn. 52), S. 14 .

58 OESCH, Europarecht Band I (Fn. 10), N935.

59 oEsCH, Working Paper (Fn. 52), S. 29. 
betrifft insbesondere den Abschluss der Bilateralen Verträge. Diese sind vor dem Hintergrund der Theorie des Brussels-Effect auf zwei verschiedene Weisen zu lesen:

Erstens: Die Verträge garantieren für die Unternehmen in der Schweiz eine - jeweils sektoriell abgesicherteTeilnahme an jenem Markt, von dem der beschriebene Effekt ausgeht. Damit verändert sich auch die Rolle der schweizerischen Volkswirtschaft als Objekt des Brussels-Effect. Der bilaterale Weg verhindert einen ungebremsten, rohen Brussels-Effect, indem er die Schweiz in den europäischen Markt integriert und damit die passive Ausgangslage der Schweiz in Bezug auf den Effect möglichst zugunsten der schweizerischen Marktteilnehmer:innen ausgestaltet. Dies geschieht durch den Abbau von Handelshemmnissen und durch geregelte Verhältnisse mit der EU, welche für die schweizerische Volkswirtschaft ein hohes Mass an Rechts- und Planungssicherheit gewährleisten. ${ }^{60}$ Die Bilateralen Verträge wirken unter dieser Sicht kanalisierend. So ist der volkswirtschaftliche Effekt einer bilateralen Kooperation mit dem EU-Markt in der Praxis beträchtlich. Einer Studie des BAK Economics zufolge würde sich das BIP ohne die bilateralen Verträge jedes Jahr graduell verringern und bis zum Jahr 2035 um 7,1 \% sinken, was einer entgangenen Wirtschaftsleistung von 630 Milliarden Franken entsprechen würde. In derselben Studie wird auch errechnet, welche individuellen ökonomischen Vorteile die bilateralen Verträge pro Kopf einbringen: Ohne die Bilateralen I würde das persönliche Einkommen in der Schweiz im Jahr 2025 um CHF 3400 tiefer liegen als unter einer Beibehaltung der Verträge. ${ }^{61}$ Eine Studie der Bertelsmann-Stiftung wiederum befasst sich mit der Frage, welche Regionen bzw. Staaten innerhalb des europäischen Binnenmarkts am meisten vom Modell des Binnenmarkts profitieren. Auch hier liegt die Schweiz als «teilintegrierte» Nation an der Spitze (und damit vor sämtlichen Mitgliedstaaten der Union); der Zugang zum Binnenmarkt sorgt gemäss dem Modell dieser Studie für eine Erhöhung des jährlichen Einkommens um 2914 Euro. ${ }^{62}$ Wie die dargelegten Zahlen klar ausdrücken, gelingt es der Schweiz unter «Zuhilfenahme» der Bilateralen Verträge weitgehend, den Brussels-Effect damit für schweizerische Unternehmen zu verringern, indem für diese Unternehmen geringere Handelshemmnisse bestehen. Das System der Bilateralen Verträge vermag es sogar, die "passive Ausgangslage» der schweizerischen Unternehmen ökonomisch in einen Mehrwert umzumünzen.

60 Vgl. EDA, Das Wichtigste in Kürze (Fn. 46).

61 Analyse Economiesuisse (Fn.32).

62 Analyse Economiesuisse (Fn.32).
Zweitens: Der Brussels-Effect führt nach der Deutung 29 BRADFORDS gemeinhin unilaterale Änderungen des Verhaltens privater Marktteilnehmer:innen herbei, wobei eine Angleichung an EU-Regulatorien beabsichtigt wird. Die Bilateralen Abkommen zeigen jedoch, dass dies nicht die einzige Form ist, in welcher der Brussels-Effect zum Tragen kommen kann: Auch klassische völkerrechtliche Verträge können Ausdruck eines extraterritorialen Drucks des EU-Rechts sein. Bezeichnet werden kann dies, gerade im Zusammenhang mit den Bilateralen Abkommen, als «Brussels-Effect suigeneris» oder «Brussels-Effect via Staatsvertrag». Wie dargelegt basiert der Abschluss der verschiedenen Bilateralen Abkommen einerseits auf der Überzeugung, eine bilaterale Annäherung an die EU sei unter dem Eindruck einer bereits tiefgehend europäisierten Rechtsordnung eher möglich. Andererseits dient die bilaterale Kooperation mit der EU auch ausdrücklich dem Ausgleich von Nachteilen, die schweizerische Unternehmen in ihrer Marktposition erleiden, weil die Schweiz nicht Teil des EU-Markts ist (bzw. war). Die Schweiz geht also auch völkerrechtliche Verpflichtungen mit der EU ein, weil die wirtschaftliche Realität diesen Schritt nahelegt. Die Bilateralen Abkommen sind also selbst sowohl Instrument zur Kontrolle bzw. Abschwächung des Brussels-Effect (wie unter 1. dargelegt), andererseits aber auch selbst das Resultat der Wirkung des gleichen Effekts.

Die Bilateralen I beabsichtigen wie dargelegt eine «schritt- 30 weise und kontrollierte gegenseitige Marktöffnung» zwischen der Schweiz und der Europäischen Union. Auch die Bilateralen II sind auf die «fortschreitende Integration der Schweiz in den Binnenmarkt unter Schaffung einer möglichst parallelen Rechtslage» ${ }^{6} \mathbf{3}$ ausgerichtet; ihre Regelungsgegenstände sind jedoch stets sektorieller Natur; die Schweiz ist in bestimmten Bereichen in den europäischen Binnenmarkt integriert. Im Bereich der vier Grundfreiheiten des EU-Binnenmarkts besteht eine weitgehende Liberalisierung; die Schweiz ist weitgehend in den freien Warenverkehr mit Industrieprodukten integriert. Im Bereich der Dienstleistungen bestehen (trotz dem Fehlen eines umfassenden Dienstleistungsabkommens ${ }^{64}$ ) verschiedene Erleichterungen im grenzüberschreitenden Bereich (insb. was kurzzeitige Erbringungen von Dienstleistungen angeht) im Freizügigkeitsabkommen vom 21.Juni 1999 (FZA). ${ }^{65}$ Das FZA erfasst auch eine weitgehende Niederlassungs- und die Arbeitnehmerfreizügigkeit für natürliche Personen. Für juristische Personen bestehen im Versicherungsabkommen und dem

63 OESCH, Working Paper (Fn. 52), S. 16.

64 OESCH, Europarecht Band I (Fn. 10), N904.

65 Abkommen zwischen der Schweizerischen Eidgenossenschaft einerseits und der Europäischen Gemeinschaft und ihren Mitgliedstaaten andererseits über die Freizügigkeit vom 21.Juni 1999 (Freizügigkeitsabkommen, FZA; SR 0.142.112.681). 
Luftverkehrsabkommen weitere Erleichterungen. Damit unterscheidet sich die Rechtsposition der Schweiz markant von verschiedenen Drittstaaten und den Marktpositionen der dort domizilierten Staaten. In anderen Bereichen, die durch keines der sektoriellen Abkommen erfasst sind, unterscheidet sich die Rechtsstellung der Schweiz jedoch kaum von derjenigen von Drittstaaten, etwa im Bereich der Agrarprodukte oder des Wettbewerbsrechts. Mit dieser sich sektoriell von der Rechtsposition anderer Drittstaaten abhebenden Situation der Schweiz geht auch eine bloss insular kanalisierte Wirkung des Brussels-Effect einher. ${ }^{66}$ Die Schweiz nimmt in begrenzten Bereichen an jenem Markt teil, von welchem die Impulse für den Brussels-Effect ausgehen. In dieser vertraglich garantierten sektoriellen Marktöffnung zum (im Sinn des Brussels-Effect) wirkenden Markt liegt nun auch die Hauptunterscheidung der Rechtsstellung der Schweiz nach Abschluss der Bilateralen von der Rechtsstellung von Drittstaaten.

31 Während sich die Position der Schweiz gegenüber der Europäischen Union unter Zuhilfenahme der BrusselsEffect-Theorie also so deuten lässt, stellt sich weiter die Frage, wie die Schweiz gegenüber Drittstaaten am Brussels-Effect teilnimmt. Beantworten lässt sich dies unter Zuhilfenahme der dargestellten Parameter des Brussels Effect. Nach BRADFORD kommt der Brussels-Effect unter der Voraussetzung des Eintritts verschiedener Faktoren zustande: der Grösse des kontrollierten Marktes, der institutionellen Kapazität zur Durchsetzung strikterer Regulatorien, einem unelastischen Ziel der Regulatorien und einer fehlenden Rentabilität der Differenzierung der Produktion. Ebenso haben die Regulatorien inhaltlich dichter bzw. strikter zu sein als die Regulierungen anderer vergleichbar attraktiver Märkte. ${ }^{67}$ Die Schweiz greift mit ihrer sektoriellen Teilnahme am EU-Binnenmarkt nun verschiedentlich in diese Faktoren ein und verstärkt sie: Einerseits vergrössert die sektorielle Integration der Schweiz den EU-Binnenmarkt um mehrere Millionen überdurchschnittlich kaufkräftiger Konsument:innen. Die in den Bilateralen Abkommen festgehaltenen Pflichten zur Durchsetzung des übernommenen EU-Besitzstandes (etwa im Bereich des Schengen-Acquis) erweitern zudem die institutionelle Kapazität der EU zur Regulierung via Staatsvertrag sektoriell auf den schweizerischen Markt. Die institutionelle Kapazität der EU zur Durchsetzung strikter Regulatorien wird in eine staatsvertragliche Pflicht «übersetzt». Die Integration der Schweiz in den europäischen Binnenmarkt durch die Bilateralen Verträge verstärkt mit anderen Worten aus der Sicht von Drittstaaten entsprechend den Brussels-Effect.

66 Vgl. zum Ganzen auch HEINEMANN (Fn. 34), S. 15 67 Vgl. Rz. $7 \mathrm{ff}$.

\section{b) Theoretische Einordnung des Inst A}

Die Idee eines institutionellen Abkommens ist mit Blick 32 auf die Ausführungen zu den Bilateralen Abkommen als Fortbildung eines sektoriellen Integrationsvorgangs zu sehen. Der auf den Brussels-Effect ausgleichend wirkende bilaterale Weg wird einerseits gesichert, andererseits aber auch vertieft, indem die Schweiz sich etwa stärker in den Gesetzgebungsprozess der Union einbringen könnte ${ }^{68}$ oder die Anordnung von wirtschaftlichen Sanktionen gegen die Schweiz unabhängigbeurteilt werden würde. ${ }^{69}$

Ein InstA, so weiterhin die Idee, würde verschiedene 33 Aspekte des sektoriellen Marktzugangs der Schweiz zu jenem Markt regeln, der als Instrument zur Ausübung extraterritorialer Macht im Sinn des Brussels-Effect agiert. Der beschriebene extraterritoriale Druck des EU-Rechts bliebe damit in einen Rahmen eingebunden, welcher Rechtssicherheit und Überschaubarkeit bietet. Ein solches Abkommen schafft für die Verträge, welche die schweizerische Volkswirtschaft in den europäischen Binnenmarkt integrieren, einheitliche Regelungen in Bezug auf anwendungsbezogene Fragen, sichert eine unabhängige Überprüfung von Sanktionen und beteiligt die Schweiz stärker am Zustandekommen des unionsrechtlichen Sekundärrechts. ${ }^{70}$ Ebenso legt es verbindliche Regelungen für die Weiterführung und den allfälligen Ausbau des bilateralen Wegs fest. Damit würde der beschriebene ausgleichende Effekt, welchen bereits die Bilateralen Verträge auf die Auswirkungen des Brussels-Effect haben, verstetigt.

Nur ein vertraglich garantierter Marktzugang sichert ab, 34 dass die wirtschaftlichen Folgen des auf die schweizerische Volkswirtschaft ausgeübten ökonomischen Drucks verkraftbar bleiben. Ein Rahmenabkommen verstetigt damit einen ökonomischen Effekt, welcher sich aus der beschriebenen sektoriellen Marktintegration ergibt.

\section{Fazit}

Für Drittstaaten stellt sich die Frage, wie mit dem Brussels- 35 Effect umzugehen ist. Je stärker die Volkswirtschaft eines Drittstaates mit dem europäischen Binnenmarkt verbunden ist, desto drängender muss diese Frage erscheinen. Wie dargelegt ist das politische Handeln der Schweiz in verschiedenerlei Hinsicht geprägt von der Grundüberzeugung, eine regulierungsbezogene Anbindung an die Union stelle eine wirtschaftliche Notwendigkeit dar. ${ }^{71}$

68 Vgl. hierzu COTTIER, Souveränität (Fn. 47), S. 345

69 Vgl. Rz. 23.

70 COTTIER, Souveränität (Fn. 47), S.367.

71 Integrationsbericht (Fn. 40); Aussenpolitischer Bericht 2000, Präsenz und Kooperation. Interessenwahrung in einer zusammenwachsenden Welt vom 15. November 2000 (BBl 2001261$).$ 
Mit Blick auf die Mittel, mit denen gemäss BRADFORDS Theorie geopolitischer Einfluss ausgeübt wird, sind denn auch Ansätze zum Abbau ökonomischer, rechtlicher und technischer Hürden via Staatsverträge und damit verbundene Harmonisierungsbemühungen für den Handel mit Marktteilnehmer:innen aus dem europäischen Binnenmarkt denkbar. Der in seiner Intensität ungewöhnliche extraterritoriale Einfluss, welchen die Union auf die Schweiz ausübt, tritt die Schweiz mit einem klassischen Handlungsinstrument der internationalen Zusammenarbeit gegenüber: dem völkerrechtlichen Vertrag.

Angesichts der aktuellen Diskussion um ein InstA macht jedoch eine explizite Einordnung mithilfe von BRADFORDS Brussels-Effect-Theorie den politischen Handlungsbedarf noch ein wenig deutlicher. Es lässt sich mit Blick auf das Gesagte festhalten, dass die Weiterführung des bilateralen Wegs unter Klärung institutioneller und rechtsanwendungsbezogener Fragen die beste Lösung ist, die faktische wie die rechtliche Position der Schweiz im Vergleich zu anderen Drittstaaten zu stärken, ${ }^{72}$ da der «bilaterale Weg» eine wirtschaftliche Teilintegration zulässt, ohne gleichzeitig «Abstriche an den Grundprinzipien des schweizerischen Staatsverständnisses» ${ }^{73}$ zu erfordern. So hätte das E-InstA die Position gegenüber der EU unter Beibehaltung der Integrationstiefe der schweizerischen Volkswirtschaft durch ein vereinheitlichtes Streitbeilegungsverfahren gestärkt, sowie einen formalisierten Prozess zur Anordnung von Ausgleichsmassnahmen und eine vertiefte Teilnahme der Schweiz am europäischen

\section{Vgl. Rz. 33.}

73 OESCH, Europarecht Band I (Fn. 10), N974.
Gesetzgebungsprozess gesichert. Eine Stärkung der Position der Schweiz angesichts der unweigerlichen extraterritorialen Wirkung des Unionsrechts lässt sich daraus ableiten. Das gilt trotz der offenen Rechtsfragen, die sich in Bezug auf das E-InstA stellen, etwa die fragliche Weitergeltung der Guillotineklausel aus den Bilateralen-IVerträgen von 1999, oder die schwerliche Voraussehbarkeit der möglichen Sanktionen, falls die Schweiz sich im Einzelfall gegen die Übernahme von EU-Recht aussprechen würde. Ebenso in die Kategorie der offenen Rechtsfragen fällt die Unklarheit, nach welchen Massstäben die in Art.10 Abs. 7 E-InstA festgehaltene Verhältnismässigkeit bei Ausgleichsmassnahmen beurteilen lässt, die sowohl durch die EU als auch durch die Schweiz ergriffen werden können, falls den vertraglichen Verpflichtungen nicht nachgekommen wird. ${ }^{74}$

Letztlich muss nach einer Analyse der Auswirkungen des 37 Brussels-Effect auf die Schweiz sowie der tiefgreifenden Folgen der schweizerischen Reaktion auf den BrusselsEffect jedoch auch festgehalten werden, dass die Schweiz ihre Rolle im europäischen Binnenmarkt und in Europa längerfristig grundsätzlich zu überdenken hat. ${ }^{75}$ Das Rahmenabkommen, so zeigt die bundesrätliche Entscheidung, ${ }^{76}$ kann hierzu nach der Ansicht der politischen Entscheidungsträger:innen keinen Abschluss bilden. Auch in dieser Frage wird BRADFORDS Theorie als Orientierungshilfe dienen können.

74 BREITENMOSER/HIRSBRUNNER (Fn. 50), S. 519.

75 Gl.M. ohne Erwähnung des Brussels-Effect OESCH, Europarecht Band I (Fn. 10), N975.

76 Vgl. Medienmitteilung des Bundesrates vom 26. Mai 2021 (Das Institutionelle Abkommen Schweiz-EU wird nicht abgeschlossen). 